\title{
Mechanisms of the anti-hypertensive effect of Hibiscus sabdariffa L. calyces
}

\author{
M. Ajay ${ }^{\text {a }}$, H.J. Chai ${ }^{\text {a }}$, A.M. Mustafa ${ }^{\text {a }}$, A.H. Gilani ${ }^{\text {b }}$, M.R. Mustafa ${ }^{\text {a,* }}$ \\ ${ }^{a}$ Department of Pharmacology, Faculty of Medicine, University of Malaya, 50603 Kuala Lumpur, Malaysia \\ ${ }^{\mathrm{b}}$ Department of Biological and Biomedical Sciences, The Aga Khan University, Karachi 74800, Pakistan
}

Received 29 December 2005; received in revised form 3 July 2006; accepted 11 August 2006

Available online 15 August 2006

\begin{abstract}
Previous studies have demonstrated the anti-hypertensive effects of Hibiscus sabdariffa L. (HS) in both humans and experimental animals. To explore the mechanisms of the anti-hypertensive effect of the HS, we examined the effects of a crude methanolic extract of the calyces of HS (HSE) on vascular reactivity in isolated aortas from spontaneously hypertensive rats. HSE relaxed, concentration-dependently, $\mathrm{KCl}$ (high $\mathrm{K}^{+}$, $80 \mathrm{mM}$ )and phenylephrine (PE, $1 \mu \mathrm{M}$ )-pre-contracted aortic rings, with a greater potency against the $\alpha_{1}$-adrenergic receptor agonist. The relaxant effect of HSE was partly dependent on the presence of a functional endothelium as the action was significantly reduced in endothelium-denuded aortic rings. Pretreatment with atropine $(1 \mu \mathrm{M})$, L-NAME $(10 \mu \mathrm{M})$ or methylene blue $(10 \mu \mathrm{M})$, but not indomethacin $(10 \mu \mathrm{M})$, significantly blocked the relaxant effects of HSE. Endothelium-dependent and -independent relaxations induced by acetylcholine and sodium nitroprusside, respectively, were significantly enhanced in aortic rings pretreated with HSE when compared to those observed in control aortic rings. The present results demonstrated that HSE has a vasodilator effect in the isolated aortic rings of hypertensive rats. These effects are probably mediated through the endothelium-derived nitric oxide-cGMP-relaxant pathway and inhibition of calcium $\left(\mathrm{Ca}^{2+}\right)$-influx into vascular smooth muscle cells. The present data further supports previous in vivo findings and the traditional use of HS as an anti-hypertensive agent.
\end{abstract}

(c) 2006 Elsevier Ireland Ltd. All rights reserved.

Keywords: Hibiscus sabdariffa L.; Endothelium; Nitric oxide; Hypertension

\section{Introduction}

Hibiscus sabdariffa Linn. (HS) (Syn: Roselle, Rozelle, Indian sorrel, Sour tea and Karkade) belonging to the Malvaceae family have long been used in indigenous system of medicine for treating hypertension (Oliver, 1960). The extracts of HS exert potent antioxidant effects in vitro, achieved via the presence of several active constituents chief among which are flavonoids and vitamins (Tseng et al., 1997; Tsai et al., 2002; Hirunpanich et al., 2005). Recent pharmacological studies have shown that HS extracts significantly reduced blood pressure in humans (HajiFaraji and Haji-Tarkhani, 1999; Herrera-Arellano et al., 2004) and in experimental animals (Ali et al., 1991; Adegunloye et al., 1996; Onyenekwe et al., 1999; Odigie et al., 2003). For example, chronic administration of the aqueous extract of HS decreased blood pressure and reduced cardiac hypertrophy in 2-kidney 1-

\footnotetext{
* Corresponding author. Tel.: +60 379674952; fax: +60 379674791.

E-mail address: rais@um.edu.my (M.R. Mustafa).
}

clip (2K-1C) rat model of hypertension (Odigie et al., 2003), and inhibited vascular tone in isolated vascular preparations including the rat thoracic aorta (Ali et al., 1991; Obiefuna et al., 1993; Adegunloye et al., 1996). However, the exact mechanisms responsible for these effects of HS are not fully understood. In addition, the possibility that the extracts of HS could produce its relaxant activities in hypertension was not investigated.

The present investigation was, therefore, undertaken to examine the possible mechanisms underlying the hypotensive effects of HS. For that purpose, we analyzed the effects of the crude methanolic extract of calyces of HS (HSE) on vasomotor responses of aortic rings isolated from spontaneously hypertensive rats (SHR).

\section{Materials and methods}

\subsection{Preparation of $H S E$}

HS calyces were obtained from a local plantation in Terengganu, Malaysia and authenticated by the Department of Botany, 
Institute of Biological Sciences, University of Malaya. The voucher specimen (No. KLU 041786) was deposited in Herbarium of the University of Malaya. The samples were washed for any contaminants, dried in oven at $50{ }^{\circ} \mathrm{C}$ and then crushed in a motar and pestle. The powdered calyces were subjected to exhaustive soxhlet extraction with methanol as extraction solvent. The extracts were then evaporated to dryness using rotary evaporator $\left(40^{\circ} \mathrm{C}\right)$ and freeze-dried. Despite the traditional practice of using the aqueous extracts of HS, we choose to extract HS with methanol as the solvent with the view to dissolve both polar and non-polar constituents, which are equally possible to represent the active compounds.

\subsection{Drugs and chemicals}

The following chemicals were obtained from the sources specified: acetylcholine chloride, atropine, indomethacin, phenylephrine, $N \omega$-nitro-L-arginine methyl ester, methylene blue (Sigma Chemicals Co., St. Louis, USA), sodium nitroprusside (SNP), Krebs salts (BDH Limited and BDH Laboratory Supplies, Poole, England) and pentobarbitone sodium (Rhone Merieux, Harlow, Essex). All drugs, except for indomethacin and HSE, were dissolved in distilled water. Indomethacin was dissolved in $0.5 \%(\mathrm{w} / \mathrm{v})$ sodium carbonate whereas freeze-dried HSE was reconstituted in 5\% (v/v) dimethylsulfoxide (DMSO). The final concentrations were prepared by serial dilutions with distilled water and the final concentration of DMSO was adjusted to less than $0.05 \%(\mathrm{v} / \mathrm{v})$, which has been shown to be devoid of any observable effects on muscle tone (Ajay et al., 2003).

\subsection{Preparation of rat thoracic aorta rings}

The animals were obtained from the University of Malaya animal unit, and all the experimental procedures utilized were performed in accordance with guidelines issued by the University of Malaya Animal Experimentation Ethics Committee. Male SHR animals, aged 20-21 weeks, with established hypertension (systolic blood pressure $\geq 175 \mathrm{mmHg}$, measured non-invasively using tail-cuff method, NIBP machine, IITC Inc., USA) were housed in an air-conditioned room $\left(24 \pm 2{ }^{\circ} \mathrm{C}\right.$ and $40 \pm 5 \%$ humidity) on a day/night cycle and supplied with standard rat chow (Gold Coin Feed Mills Sdn. Bhd., Malaysia) and tap water ad libitium. The rats were anesthetized with pentobarbitone sodium $(60 \mathrm{mg} / \mathrm{kg}$ of body weight, IP) and the thoracic aorta was isolated, cleaned of fat and connective adipose tissue, and cut into $3-5 \mathrm{~mm}$ long rings. The rings were suspended horizontally in tissue chambers containing $5 \mathrm{~mL}$ of Krebs physiological solution (KPS) of following composition (in $\mathrm{mM}$ ): $\mathrm{NaCl} 118.2$, $\mathrm{KCl} 4.7, \mathrm{CaCl}_{2} \cdot 2 \mathrm{H}_{2} \mathrm{O} 2.5, \mathrm{MgSO}_{4} 1.2, \mathrm{KH}_{2} \mathrm{PO}_{4} 1.2$, glucose 11.7, $\mathrm{NaHCO}_{3}$ 25.0, and EDTA 0.026. Special care was taken to avoid damage to the endothelium. The tissue-bath solution was aerated continuously with $95 \% \mathrm{O}_{2}-5 \% \mathrm{CO}_{2}$ at $37^{\circ} \mathrm{C}$. Aortic rings were allowed to equilibrate at an optimal tension of $1 \mathrm{~g}$ for $45 \mathrm{~min}$; during this period Krebs solution was replaced every $15 \mathrm{~min}$ and, if needed, the tension was readjusted to $1 \mathrm{~g}$. Before starting actual experimental protocol, contractile responsiveness of the aortic rings was tested by repeated exposure to $\mathrm{KCl}$ solu- tion (high $\mathrm{K}^{+}, 80 \mathrm{mM}$ ). Responses were recorded isometrically via a force-displacement transducer connected to a Mac Lab recording system (AD Instruments, Australia) equipped with a portable display monitor.

\subsection{Pharmacological studies}

Following the initial equilibration, to investigate a possible relaxant action of the HS, the aortic rings were pre-contacted with either high $\mathrm{K}^{+}$- or phenylephrine (PE, $\left.1 \mu \mathrm{M}\right)$, and the relaxant effects to HSE at different concentrations (from $10 \mathrm{ng} / \mathrm{mL}$ to $1 \mathrm{mg} / \mathrm{mL}$ ) were recorded by adding cumulative concentrations of HSE to the tissue bath at $3 \mathrm{~min}$ intervals between successive concentrations. To confirm the dependence of relaxant effects of HSE on endothelium, relaxations to HSE against $\mathrm{PE}(1 \mu \mathrm{M})$-induced contractions are performed in endotheliumdenuded aortic rings. To investigate the involvement of EDNO and prostacyclin $\left(\mathrm{PGI}_{2}\right)$, aortic rings were pre-incubated with $N \omega$-nitro-L-arginine methyl ester (L-NAME, $10 \mu \mathrm{M}$ ), an EDNO synthase inhibitor, and indomethacin $(10 \mu \mathrm{M})$, an cyclooxygenase inhibitor, respectively, and the relaxations to HSE was then performed. To examine possible participation of cholinergic mechanisms, relaxations to HSE were performed in aortic rings pre-incubated with atropine $(1 \mu \mathrm{M})$, a competitive muscarinic antagonist. Lastly, to characterize possible contribution of EDNO/cGMP-relaxant pathway, relaxations to HSE are performed in aortic rings pre-incubated with methylene blue $(10 \mu \mathrm{M})$, a cGMP-inhibitor. To examine the effects of various pharmacological agents on relaxations to HSE, various agents were added to the organ bath 20 min before the addition of PE $(1 \mu \mathrm{M})$ and relaxations to HSE were performed in their continued presence. In experiments using endothelium-denuded aortic rings, endothelium was removed by gentle rubbing of intimal surface of aortic rings with a blunted forceps, and denudation of endothelium was confirmed by absence of relaxations $(<5-10 \%)$ to acetylcholine $(10 \mu \mathrm{M})$.

In a separate set of experiments, the effects of HS on the responses of aortic rings to endothelium-dependent and independent vasodilators acetylcholine ( $\mathrm{ACh}$ ) and sodium nitroprusside (SNP), respectively, were examined. In these experiments, the concentration-response curves for $\mathrm{ACh}$ (from $1 \mathrm{nM}$ to $10 \mu \mathrm{M}$ ) and SNP (from $10 \mathrm{pM}$ to $1 \mu \mathrm{M}$ ) were recorded in PE $(1 \mu \mathrm{M})$ pre-contracted aortic rings incubated with HSE $(0.3 \mathrm{mg} / \mathrm{mL})$ or with its vehicle, which served as control. HSE or its vehicle was added to the organ bath $20 \mathrm{~min}$ before addition of $\mathrm{PE}$ and relaxations to $\mathrm{ACh}$ and SNP were performed in their continued presence. ACh and SNP were added cumulatively to the tissue bath at peak of the PE-induced contraction with $3 \mathrm{~min}$ intervals between successive concentrations.

\subsection{Data presentation and statistical analysis}

The concentrations mentioned in the text or in the figures represent the final tissue-bath concentrations. All values are given as mean \pm S.E.M. and ' $n$ ' indicates number of determinations performed under respective experimental conditions. 
Relaxant responses to HSE, ACh, and SNP are expressed as the relative percentage reduction of the respective contraction. The responses were analyzed using a computer software (Prism version 2.0, Graphpad software, USA) and $R_{\max }$ (response at highest concentration of respective agonist tested) and $\mathrm{pEC}_{50}$ ( $-\log$ of effective median concentration) values were calculated. Significant differences were tested using Student's $t$-test or oneway analysis of variance (ANOVA) and $p$ values $<0.05$ were considered significant.

\section{Results}

\subsection{Relaxant effects of HSE on high $\mathrm{K}^{+}$- and PE-pre-contracted aortas}

High $\mathrm{K}^{+}(80 \mathrm{mM})$ - and PE $(1 \mu \mathrm{M})$-induced sustained contractions in the isolated aorta, reaching a maximum tension of (in grams) of $0.99 \pm 0.08$ and $0.90 \pm 0.08$, respectively. Cumulative addition of HSE to the bath inhibited, concentration-dependently, these sustained contractions with a maximum relaxation of $27.9 \pm 4.36 \%\left(\mathrm{pEC}_{50}=3.37 \pm 0.26\right)$ and $86.01 \pm 4.84 \%\left(\mathrm{pEC}_{50}=3.83 \pm 0.18\right)$ reduction in the high $\mathrm{K}^{+}$- and PE-induced contractions, respectively (Fig. 1). At all the concentrations tested, HSE caused a significantly higher relaxant effect against PE-induced than high $\mathrm{K}^{+}$-induced contractions. The maximal relaxations to HSE against PE-induced contractions in the presence and absence of the various pharmacological interventions are given in Table 1. Removal of the endothelium significantly attenuated the vasorelaxant responses to all the concentrations of HSE (Fig. 2). Pretreatment with L-NAME $(10 \mu \mathrm{M})$, but not with indomethacin $(10 \mu \mathrm{M})$, significantly inhibited vasorelaxant responses to HSE (Fig. 3). In addition, L-NAME attenuation of HSE-induced relaxations was found to be quantitatively similar to that observed in endothelium-denuded aortic rings. Pretreatment with atropine $(1 \mu \mathrm{M})$ significantly inhibited relaxations observed at the highest concentration of HSE, but had no effect on the relaxations observed at the lower concentrations of HSE (Fig. 3). Pretreatment with methylene blue $(10 \mu \mathrm{M})$ significantly inhibited relaxations observed at all the concentrations of HSE (Fig. 3), and that this attenuation was found to be essentially similar to

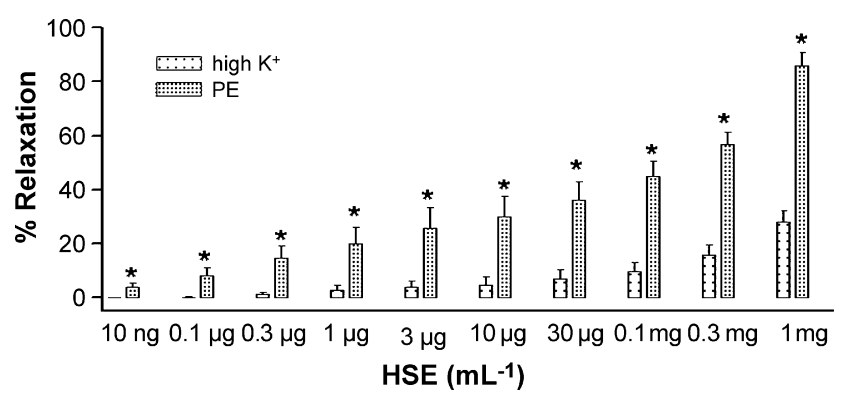

Fig. 1. Effects of methanolic extract of calyces of Hibiscus sabdariffa L. (HSE) against $\mathrm{KCl}$ (high $\mathrm{K}^{+}, 80 \mathrm{mM}$ ) and phenylephrine (PE, $1 \mu \mathrm{M}$ )-induced contractions in endothelium-intact aortic rings isolated from spontaneously hypertensive rats. Relaxation was calculated as \% reduction in the respective agonistinduced contraction. Symbols represent mean \pm S.E.M. $(n=6$ or 7$) .{ }^{*} p<0.05$ vs. high $\mathrm{K}^{+}$pre-contracted aortic rings.
Table 1

Maximal relaxations to methanolic extract of calyces of Hibiscus sabdariffa L. (HSE) against phenylephrine (PE, $1 \mu \mathrm{M}$ )-induced contractions in isolated aortic rings from spontaneously hypertensive rats under various experimental conditions

\begin{tabular}{ll}
\hline Treatment group & $\begin{array}{l}\text { Maximal relaxation to HSE }(\% \\
\text { reduction of PE contraction) }\end{array}$ \\
\hline Control (untreated) & $86.01 \pm 4.84$ \\
L-NAME $(10 \mu \mathrm{M})$ & $53.25 \pm 4.77^{*}$ \\
Indomethacin $(10 \mu \mathrm{M})$ & $75.58 \pm 5.56$ \\
Atropine $(10 \mu \mathrm{M})$ & $67.00 \pm 5.36^{*}$ \\
Methylene blue $(10 \mu \mathrm{M})$ & $46.79 \pm 1.96^{*}$ \\
Endothelium-denuded & $54.79 \pm 9.76^{*}$ \\
\hline
\end{tabular}

Values represent mean \pm S.E.M. of four to seven determinations. ${ }^{*} p<0.05$ vs. control aortic rings.

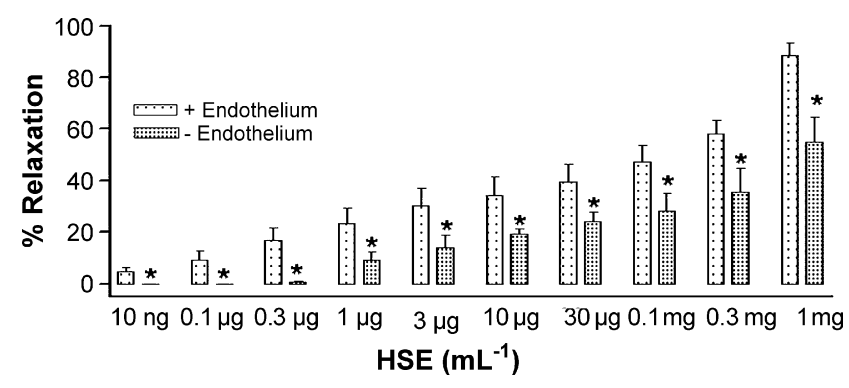

Fig. 2. Effects of methanolic extract of calyces of Hibiscus sabdariffa L. (HSE) against phenylephrine (PE, $1 \mu \mathrm{M})$-induced contractions in endothelium-intact (+endothelium) and -denuded (-endothelium) aortic rings isolated from spontaneously hypertensive rats. Relaxation was calculated as \% reduction in the respective contraction. Symbols represent mean \pm S.E.M. $(n=6$ or 7 for intact rings, 4 or 5 for denuded rings). ${ }^{*} p<0.05$ vs. equivalent response in endotheliumintact aortic rings.

that observed in endothelium-intact aortic rings pre-incubated with L-NAME and also in endothelium-denuded aortic rings.

\subsection{Effects of HSE on ACh- and SNP-induced aortic relaxations}

PE $(1 \mu \mathrm{M})$-induced increases in the tone with the same magnitude in aortic rings pre-incubated for 20 min either with

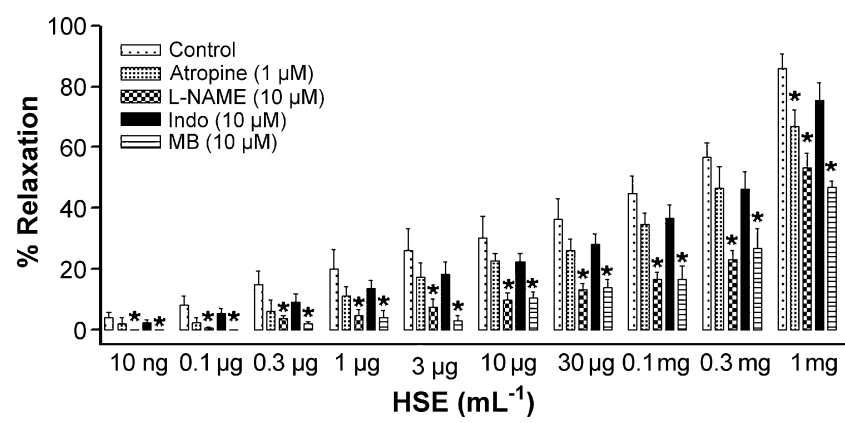

Fig. 3. Effects of methanolic extract of calyces of Hibiscus sabdariffa L. (HSE) against phenylephrine (PE, $1 \mu \mathrm{M}$ )-induced contractions in endothelium-intact aortic rings incubated with atropine $(1 \mu \mathrm{M})$, L-NAME $(10 \mu \mathrm{M})$, indomethacin (Indo, $10 \mu \mathrm{M}$ ) or methylene blue (MB, $10 \mu \mathrm{M})$. Relaxation was calculated as \% reduction in the respective contraction. Symbols represent mean \pm S.E.M. $(n=6$ or 7 for control, 4 or 5 in the presence of interventions). ${ }^{*} p<0.05$ vs. equivalent response in control aortic rings. 


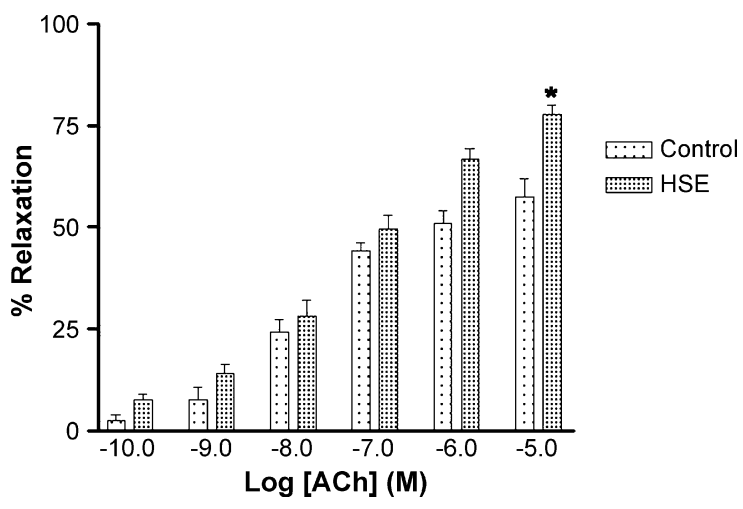

Fig. 4. Relaxation responses to endothelium-derived nitric oxide (EDNO)mediated vasodilator acetylcholine $(\mathrm{ACh})$ in spontaneously hypertensive rat aortic rings pre-incubated with either methanolic extract of calyces of Hibiscus sabdariffa L. (HSE, $0.3 \mathrm{mg} \mathrm{mL}^{-1}$ ) or with its vehicle, which served as control. The aortic rings were pre-contracted with phenylephrine $(1 \mu \mathrm{M})$ and the relaxation was calculated as \% reduction in respective contraction. Symbols represent mean \pm S.E.M. of six or seven determinations. ${ }^{*} p<0.05$ vs. control.

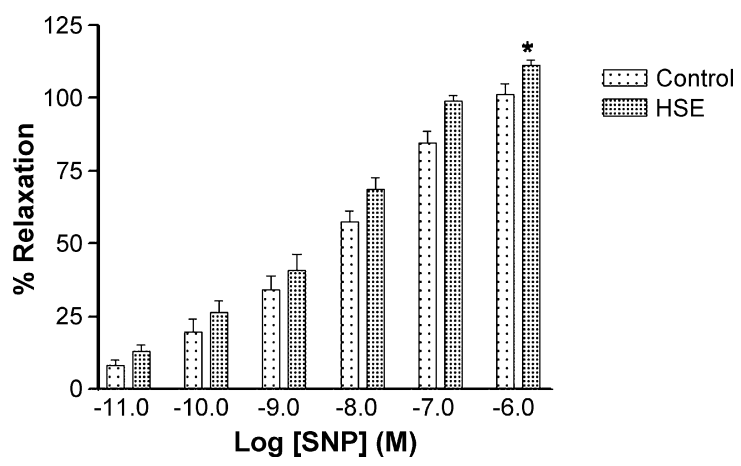

Fig. 5. Relaxation responses to non-endothelium-derived nitric oxide (EDNO)mediated vasodilator sodium nitroprusside (SNP) in spontaneously hypertensive rat aortic rings pre-incubated with either methanolic extract of calyces of Hibiscus sabdariffa L. (HSE, $0.3 \mathrm{mg} \mathrm{mL}^{-1}$ ) or its vehicle, which served as control The aortic rings were pre-contracted with phenylephrine $(1 \mu \mathrm{M})$ and the relaxation was calculated as \% reduction in respective contraction. Symbols represent mean \pm S.E.M. of six or seven determinations. ${ }^{*} p<0.05$ vs. control.

HSE $(0.3 \mathrm{mg} / \mathrm{mL})$ or its vehicle, which served as control, reaching a maximum tension of (in grams) $0.78 \pm 0.04$ and $0.89 \pm 0.08$, respectively. This suggests that pretreatment of aortas with HSE showed no significant effect on PEinduced contractions. Cumulative addition of ACh (Fig. 4) and SNP (Fig. 5) concentration-dependently relaxed the PE pre-contracted aortic rings with a significantly higher maximal response in HSE-pretreated (ACh: $R_{\max }=77.89 \pm 2.17 \%$, $\mathrm{pEC}_{50}=7.79 \pm 0.17 ; \mathrm{SNP}: R_{\max }=111.46 \pm 1.52 \%, \mathrm{pEC}_{50}=$ $8.09 \pm 0.20)$ aortic rings when compared to respective responses observed in vehicle-pretreated control aortic rings (ACh: $R_{\max }=57.56 \pm 4.29 \%, \quad \mathrm{pEC}_{50}=7.24 \pm 0.18 ; \quad$ SNP: $\quad R_{\max }=$ $101.1 \pm 3.84 \%, \mathrm{pEC}_{50}=7.90 \pm 0.32$ ).

\section{Discussion}

The aim of the present work was to investigate the possible mechanism(s) involved in the anti-hypertensive effects of HS using isolated aortic rings from spontaneously hypertensive rats.
The results showed that the methanolic extract of calyces of HS (HSE) relaxed, concentration-dependently, the aortic rings precontracted with $\mathrm{KCl}$ (high $\mathrm{K}^{+}, 80 \mathrm{mM}$ ) and phenylephrine (PE, $1 \mu \mathrm{M})$, but with a significantly greater relaxant effect against the $\alpha_{1}$-adrenergic receptor agonist. The relaxant effect of HSE against PE-induced contraction was partially but significantly inhibited following removal of endothelium and in the presence of atropine, L-NAME or methylene blue. Additionally, pretreatment with HSE significantly improved the vasodilator responses of the aortic rings to the endothelium-dependent and -independent vasodilators acetylcholine $(\mathrm{ACh})$ and sodium nitroprusside (SNP), respectively.

High $\mathrm{K}^{+}$and PE-induced increases in aortic tone by stimulating $\mathrm{Ca}^{2+}$ influx through voltage and receptor operated $\mathrm{Ca}^{2+}$ channels (Bolton, 1979; Karaki and Weiss, 1984). The present results demonstrated that HSE concentrationdependently relaxed the contractions to high $\mathrm{K}^{+}$and PE suggesting that it may have inhibited $\mathrm{Ca}^{2+}$ influx. This observation was consistent with a previous study, which reported an inhibition of $\mathrm{Ca}^{2+}$-influx in the relaxant effects of HS in physiological rat aortic rings (Obiefuna et al., 1993). We also found that the relaxations to HSE against PE-induced contractions were significantly higher in magnitude compared to those observed against $\mathrm{KCl}$-induced depolarization-mediated contractions. The vasoconstrictor effect of $\alpha_{1}$-adrenoceptor agonists can be modulated by endothelium-derived relaxant factors that include endothelium-derived nitric oxide (EDNO) and prostacyclin $\left(\mathrm{PGI}_{2}\right)$ (Godfraind et al., 1985). The present results demonstrated that chemical inhibition of EDNO synthesis/release by blocking endothelial nitric oxide synthase (eNOS) activity with L-NAME significantly attenuated the HSEinduced relaxations. In addition, removal of endothelium attenuated the relaxations to HSE to the same magnitude as in the L-NAME pretreated aortic rings. Furthermore, blockade of cyclooxygenase activity and thereby inhibition of $\mathrm{PGI}_{2}$ production with indomethacin was without any significant effect on HSE-induced relaxations. Taken together, these findings suggest that the relaxant effect of HSE against PE-induced contractions was partially endothelium-dependent and possibly mediated by EDNO-dependent actions.

To further investigate the mechanisms of EDNO synthesis/release and subsequent relaxation of vascular smooth muscle cells, we examined the relaxant effects to HSE in aortic rings incubated with atropine, a competitive muscarinic antagonist, or methylene blue, a cGMP inhibitor. In the presence of atropine, relaxations to HSE were only blocked at the highest concentration of the extract tested. This attenuation was of a significantly lesser magnitude compared to that observed with L-NAME. These findings suggested the involvement of mechanism(s) other than or in addition to activation of muscarinic receptors (i.e., cholinergic activation) in the HSE-induced synthesis/release of EDNO. It was reported that EDNO-induced relaxation was due to an increase of cGMP levels in vascular smooth muscle cells in response to activation of guanylate cyclase (Hansen and Nedergaard, 1999). In the present study, HSE-induced relaxations were significantly inhibited following inhibition of cGMP synthesis with methylene blue. This atten- 
uation was quantitatively similar to that observed in aortic rings accounted for inhibition of eNOS activity with L-NAME. This data suggests that the relaxant effects of HSE involved activation of EDNO/cGMP-relaxant pathway activity in addition to the increased synthesis/release of EDNO.

Acetylcholine stimulates the muscarinic receptor, which increases intracellular $\mathrm{Ca}^{2+}$ in endothelial cells leading to the synthesis/release of EDNO. EDNO diffuses into adjacent smooth muscle cells and activates cGMP production to cause relaxation of vascular smooth muscle cells. A reduced EDNOmediated vasodilator response to acetylcholine is consistently documented in various hypertensive vessels including rat thoracic aortas (Taddei et al., 1998; Gewaltig and Kojda, 2002; Ajay et al., 2006). The exact mechanism underlying this attenuation is not fully understood. However, decreased bioavailability as well as bioactivity (i.e., reduced EDNO/cGMP-relaxant pathway activity) of EDNO coupled to reduced synthesis/release from endothelium or an increased production of superoxide anions and its reactive metabolites has been mostly adduced (Lassegue and Griendling, 2004). In the present study, pretreatment with HSE significantly improved acetylcholine-induced relaxations. Extracts of HS has been previously shown to exert potent free radical scavenging and antioxidant actions (Tseng et al., 1997; Tsai et al., 2002). Indeed, several studies have reported improved endothelium-dependent relaxations in hypertensive vessels with short-term in vitro and long-term in vivo antioxidant interventions, thus suggesting a pathological role for oxygen-derived free radicals in the impaired vascular responses in hypertension (Akpaffiong and Taylor, 1998; Ajay et al., 2006). In tandem with these reports, the enhancement in relaxations to ACh by HSE may be attributed to the preservation of EDNO from being inactivated by superoxide anions and its reactive oxygen metabolites. However, these assumptions require further confirmation. In addition, we also found that HSE also significantly improved the non-EDNO-mediated relaxations of aortas to the exogenous nitric oxide donor, SNP. SNP breaks down spontaneously to yield nitric oxide, thereby causing endothelium-independent vasodilatation by the same effector mechanism as nitric oxide released from endothelium. Taken together, these findings indicated that the improvement of vascular relaxations to acetylcholine by HSE could be due to similar mechanisms to those observed with its endothelium-dependent vasodilator effects, i.e. enhanced EDNO/cGMP-relaxant pathway activity.

Lastly, we also found that HSE relaxed aortic rings precontracted with PE; whereas, pretreatment of the rings with the extract was without any significant effect on the contractions induced by PE. This suggests that the possible involvement of $\alpha_{1}$-receptor antagonism in the effects of HSE appears unlikely. In addition, the above observation also suggests that HSE did not modify the basal release of EDNO or EDNO/cGMP-relaxant pathway activity in aortic rings isolated from spontaneously hypertensive rats.

In summary, the present study demonstrated that the methanolic extract of calyces of Hibiscus sabdariffa L. induced a vasodilator effect in isolated aortas from spontaneously hypertensive rats via endothelium-dependent and -independent vasodilator pathways. Endothelium-dependent vasodilator com- ponent results through activation of endothelium-derived nitric oxide/cGMP-relaxant pathway whereas endotheliumindependent component could be due to inhibition of $\mathrm{Ca}^{2+}$ influx. The present findings explains the blood pressure lowering effect of Hibiscus sabdariffa L. in vivo, and provided further evidence to the traditional use of the plant as an antihypertensive agent.

\section{Acknowledgement}

This work was supported by a grant (IRPA 0602031010) from the Ministry of Science, Technology and Innovation, Malaysia.

\section{References}

Adegunloye, B.J., Omoniyi, J.O., Owolabi, O.A., Ajagbona, O.P., Sofola, O.A., Coker, H.A., 1996. Mechanisms of blood pressure lowering effects of the calyx extract of Hibiscus sabdariffa in rats. African Journal of Medicine and Medical Sciences 25, 235-238.

Ajay, M., Gilani, A.H., Mustafa, M.R., 2003. Effects of flavonoids on vascular smooth muscle of the isolated rat thoracic aorta. Life Sciences 74, 603-612.

Ajay, M., Achike, F.I., Mustafa, A.M., Mustafa, M.R., 2006. Direct effects of quercetin on impaired reactivity of spontaneously hypertensive rat aortae. Comparative study with ascorbic acid. Clinical and Experimental Pharmacology and Physiology 33, 345-350.

Akpaffiong, M.J., Taylor, A.A., 1998. Antihypertensive and vasodilator actions of antioxidants in spontaneously hypertensive rats. American Journal of Hypertension 11, 1450-1460.

Ali, M.B., Salih, W.M., Mohamed, A.H., Homeida, A.M., 1991. Investigation of the antispasmodic potential of Hibiscus sabdariffa calyces. Journal of Ethnopharmacology 31, 249-257.

Bolton, T.B., 1979. Mechanism of action of transmitters and other substances on smooth muscles. Physiological Reviews 59, 606-718.

Gewaltig, M.T., Kojda, G., 2002. Vasoprotection by nitric oxide: mechanisms and therapeutic potential. Cardiovascular Research 55, 250-260.

Godfraind, T., Egleme, C., Osachie, A.I., 1985. Role of endothelium in the contractile response of rat aorta to alpha-adrenoceptor agonists. Clinical Science 68, 65s-71s

Haji-Faraji, M., Haji-Tarkhani, A., 1999. The effect of sour tea (Hibiscus sabdariffa) on essential hypertension. Journal of Ethnopharmacology 65, 231-236.

Hansen, K., Nedergaard, O.A., 1999. Methodologic aspects of acetylcholineevoked relaxation of rabbit aorta. Journal of Pharmacology and Toxicology 41, 153-159.

Herrera-Arellano, A., Flores-Romero, S., Chavez-Soto, M.A., Tortoriello, J., 2004. Effectiveness and tolerability of a standardized extract from Hibiscus sabdariffa in patients with mild to moderate hypertension: a controlled and randomized clinical trial. Phytomedicine 11, 375-382.

Hirunpanich, V., Utaipat, A., Morales, N.P., Bunyapraphatsara, N., Sato, H., Herunsalee, A., Suthisisang, C., 2005. Antioxidant effects of aqueous extracts from dried calyx of Hibiscus sabdariffa LINN. (Roselle) in vitro using rat low-density lipoprotein (LDL). Biological and Pharmaceutical Bulletin 28, 481-484.

Karaki, H., Weiss, G.B., 1984. Calcium channels in smooth muscle. Gastroenterology 87, 960-970.

Lassegue, B., Griendling, K.K., 2004. Reactive oxygen species in hypertension. American Journal of Hypertension 17, 852-860.

Obiefuna, P.C.M., Owolabi, O.A., Adegunloye, B.J., Obiefuna, I.P., Sofola, O.A., 1993. The petal extract of Hibiscus sabdariffa produces relaxation of isolated rat aorta. International Journal of Pharmacognosy 32, 69-74.

Odigie, I.P., Ettarh, R.R., Adigun, S.A., 2003. Chronic administration of aqueous extract of Hibiscus sabdariffa attenuates hypertension and reverses cardiac hypertrophy in $2 \mathrm{~K}-1 \mathrm{C}$ hypertensive rats. Journal of Ethnopharmacology 86, 181-185. 
Oliver, B., 1960. Medicinal plants in Nigeria. Being a Course of Four Lectures Delivered in April 1959 in the Pharmacy Department of the Nigerian College of Arts, Science and Technology, Ibadan, pp. 16-42.

Onyenekwe, P.C., Ajani, E.O., Ameh, D.A., Gamaniel, K.S., 1999. Antihypertensive effect of roselle (Hibiscus sabdariffa) calyx infusion in spontaneously hypertensive rats and a comparison of its toxicity with that in Wistar rats. Cell Biochemistry and Function 17, 199-206.

Taddei, S., Virdis, A., Ghiadoni, L., Magagna, A., Salvetti, A., 1998. Vitamin $\mathrm{C}$ improves endothelium-dependent vasodilation by restoring nitric oxide activity in essential hypertension. Circulation 97, 22222229.

Tsai, P.J., McIntosh, J., Pearce, P., Camden, B., Jordan, B.R., 2002. Anthocyanin and antioxidant capacity in Roselle (Hibiscus sabdariffa L) extract. Food Research International 35, 351-356.

Tseng, T.H., Kao, E.S., Chu, C.Y., Chou, F.P., Lin Wu, H.W., Wang, C.J., 1997. Protective effects of dried flower extracts of Hibiscus sabdariffa L. against oxidative stress in rat primary hepatocytes. Food and Chemical Toxicology $35,1159-1164$ 